\title{
INCLUSÃO DO ACOMPANHANTE NA ROTINA DE ASSISTÊNCIA AO PARTO DE ALTO RISCO
}

\author{
Inclusion of the Companion in the High-risk Delivery Care Routine
}

Letícia Natacha Bernardy ${ }^{1}$ Fabiana Fontana Medeiros ${ }^{2}$

Thais da Silva Capello ${ }^{3}$

Izabel Dayana de Lemos Santos ${ }^{4}$

Alexandrina Aparecida Maciel Cardelli ${ }^{5}$

Cátia Campaner Ferrari Bernardy ${ }^{6}$

\begin{abstract}
Resumo: Objetivo: identificar a inclusão do acompanhante no parto de alto risco. Método: trata-se de um estudo quantitativo, transversal descritivo. A população do estudo foi constituída por 319 mulheres no período pós-parto internadas em uma maternidade pública de alto risco. A pesquisa foi realizada no período de outubro de 2016 a agosto de 2017. Os dados foram compilados no programa SPSS ${ }^{\circledR}$ versão 20.0 Resultados: o estudo mostrou que grande parte $(60,8 \%)$ das mulheres foram submetidas à cesariana e foram informadas sobre o direito ao acompanhante (62,6\%). O momento da informação ofertada 37,3\% receberam na internação, $17,8 \%$ no momento do parto e $7,5 \%$ no puerpério; $37,9 \%$ das mulheres não tiveram acompanhante no momento do parto, com número expressivo $(23,2 \%)$ decorrente de dificuldades na rotina hospitalar. Conclusão: Nota-se dificuldades para incorporar o acompanhante na rotina e no atendimento ao trabalho de parto, parto e puerpério de mulheres classificadas como de alto risco.
\end{abstract}

Palavras-chaves: Parto normal; Trabalho de parto; Humanização da assistência; Saúde da Mulher; Enfermagem Obstétrica; Gravidez de Alto Risco.

\footnotetext{
${ }^{1}$ Enfermeira. Pós-graduanda de enfermagem obstétrica no Centro Universitário Filadélfia UniFil, Londrina, Paraná, Brasil. $₫$ lele_bernardy@hotmail.com. (B) https://orcid.org/0000-0002-9952-4466 http://lattes.cnpq.br/0885203257422257.

2 Enfermeira. Doutoranda em Enfermagem. Universidade Estadual de Londrina, Paraná, Brasil. $\square$ fontana.fabi@hotmail.com. Bhttps://orcid.org/0000-0002-7876-572X / http://lattes.cnpq.br/8124058784724075. 3 Enfermeira. Mestranda em Enfermagem. Universidade Estadual de Londrina, Paraná, Brasil. $\square$ capellothais@ outlook.com. Đ https://orcid.org/0000-0001-6153-2041 / http://lattes.cnpq.br/9173390740340657. 4 Enfermeira. Doutoranda em Enfermagem. Universidade Estadual de Londrina, Paraná, Brasil. $\square$ izabellemos87@hotmail.com. http://lattes.cnpq.br/7941300744615926. https://orcid.org/0000-0003-3559-5350

${ }_{5}^{5}$ Enfermeira. Doutora, Associada do Departamento de Enfermagem. Área da Saúde da Mulher e Gênero. Centro de Ciências da Saúde, Universidade Estadual de Londrina, Paraná, Brasil. $\triangle$ macielalexandrina@gmail.com. https://orcid.org/0000-0002-0222-8821 / http://lattes.cnpq.br/3592898566705036.

${ }^{6}$ Enfermeira. Doutora, Adjunta do Departamento de Enfermagem. Área da Saúde da Mulher e Gênero. Centro de Ciências da Saúde, Universidade Estadual de Londrina, Paraná, Brasil. $\square$ ccfbernardy@gmail.com. https://orcid.org/0000-0001-9723-1857 / http://lattes.cnpq.br/6127307314819853.
}

$\begin{array}{ccc}\text { Recebido em } & \text { Aceito em } & \text { Publicado em } \\ 14 / 05 / 2021 & 21 / 07 / 2021 & 03 / 09 / 2021\end{array}$


Abstract: Objective: Objective: to identify the inclusion of the companion in high-risk childbirth. Method: this is a quantitative, descriptive cross-sectional study. The study population consisted of 319 postpartum women hospitalized in a high-risk public maternity hospital. The survey was conducted from October 2016 to August 2017. Data were compiled in the SPSS ${ }^{\circledR}$ version 20.0 program Results: the study showed that a large part (60.8\%) of women underwent cesarean section and were informed about the right to a companion $(62.6 \%)$. The moment of information offered $37.3 \%$ received it during hospitalization, $17.8 \%$ at the time of delivery and $7.5 \%$ in the puerperium; $37.9 \%$ of the women did not have a companion at the time of delivery, with a significant number $(23.2 \%)$ due to difficulties in the hospital routine. Conclusion: There are difficulties in incorporating the companion into the routine and care of labor, delivery and puerperium for women classified as high risk.

Keywords: Normal birth; Labor; Humanization of care; Women's Health; Obstetric Nursing; Pregnancy, High-Risk.

\section{INTRODUÇÃO}

Para a mulher, o momento do parto é algo singular, independentemente do número de gestações, sendo cada uma, uma nova experiência. É um acontecimento de extrema importância para a mulher e sua família ${ }^{1}$.

As boas práticas de atenção ao parto destacam-se no alcance do nascimento por proporcionarem uma experiência satisfatória e positiva para a mulher e sua família. Neste sentido, quando o profissional de saúde inclui o acompanhante escolhido pela mulher durante o parto e puerpério, contribui para a confiança da parturiente, além de favorecer a humanização da assistência ${ }^{2}$.

Pensando nas boas práticas do parto, com base nas recomendações da Organização Mundial da Saúde (OMS), em 2005, foi publicada a lei 11.108, a qual garante o direito às parturientes a presença do acompanhante, de sua escolha, no trabalho de parto, parto e puerpério. Os hospitais devem manter um aviso e informar à parturiente sobre este direito, assim que ela chegue para internação. O Ministério da Saúde reconhece os benefícios que a presença do acompanhante traz à mulher, pois ela sente-se mais tranquila e segura, durante o processo, colaborando para uma experiência positiva ${ }^{3}$. A satisfação da mulher no parto está associada ao ambiente acolhedor e à presença de uma companhia, fatores que oferecem suporte emocional e físico, proporcionando sentimentos positivos para a parturiente ${ }^{4}$.

O acompanhante pode participar de todo processo de parturição. Essa pessoa deve receber informações necessárias quanto à maneira de se comportar, para que possa trazer benefícios para a mulher. Os profissionais de saúde podem realizar orientações aos 
acompanhantes, quanto a métodos não farmacológicos de alívio da dor tais como massagem, exercícios respiratórios, a prática do diálogo e o estímulo à deambulação ${ }^{5}$.

O apoio contínuo do acompanhante durante o trabalho de parto e parto favorece a redução de intervenções desnecessárias. Ao acolher o acompanhante, este pode ser estimulado ao desenvolvimento às ações de conforto físico, participando dos métodos nãofarmacológicos, e exercendo o papel de intermediário sobre os desejos da mulher com os profissionais de saúde ${ }^{6}$.

Mediante os benefícios que o acompanhante apresenta no processo de trabalho de parto e parto, surgiu a inquietação de conhecer como a inclusão do acompanhante acontece na conclusão de uma gestação de alto risco. Deste modo, este estudo teve por objetivo identificar a inclusão do acompanhante no parto de alto risco.

\section{MÉTODOS}

Trata-se de uma pesquisa descritiva, transversal, realizada em uma maternidade pública do Sul do Brasil, a qual é referência para assistência à intercorrências obstétricas e parto de alta complexidade. A pesquisa foi realizada no período de outubro de 2016 a agosto de 2017.

A amostra foi definida com base nos atendimentos da instituição um ano antes da pesquisa, utilizando ferramentas estatísticas para sua definição, utilizando fórmula de Barbeta 20127, sendo definido a amostra de 319 mulheres, no período pós-parto, internadas na maternidade. Os critérios de inclusão foram estar no pós-parto e ser classificada como gestação de alto risco segundo os critérios de near miss materno do Ministério da Saúde ${ }^{8}$. Os critérios de exclusão foram mulheres com comprometimento cognitivo ao qual impossibilitasse a realização da entrevista.

Foram realizadas consultas e transcrições dos dados da carteira de pré-natal e prontuário hospitalar, seguidas por entrevistas com as mulheres no momento do puerpério imediato, utilizando um formulário semiestruturado, realizadas diariamente na maternidade. A coleta de dados foi realizada pelos próprios pesquisadores do estudo, os quais apresentam formação strictu sensu e experiência com estudo quantitativo

As variáveis estudadas foram: faixa etária; cor de pele; situação conjugal; residência -categorizada em zona rural e urbana; escolaridade; renda familiar - categorizada 
conforme o salário mínimo do ano de 2017 (R \$ 937,00); número de gestações; número de partos normais; número de cesárea; intervalo interpartal; tipo de parto; local de parto; informações sobre o direito ao acompanhante; acompanhante presente no parto; sendo a variável por que não teve acompanhante categorizada em: dificuldades em relação a dinâmica familiar, intercorrências maternas e/ou neonatais, dificuldades em relação a rotina hospitalar e não foi informado o motivo.

Os dados foram digitados e analisados com estatística descritiva, frequência absoluta e relativa, no software IBM SPSS Statistics 20.

O estudo foi aprovado pelo Comitê de Ética em Pesquisa da Universidade Estadual de Londrina com número do parecer 1.757.596 e CAAE 59935716.7.0000.5231. As participantes foram esclarecidas sobre os objetivos da pesquisa e as que concordaram em participar do estudo assinaram o Termo de Consentimento Livre e Esclarecido em duas vias, permanecendo uma via com a participante e a outra com o pesquisador.

\section{RESULTADOS}

A faixa etária das mulheres variou entre 19 e 29 anos (48,2\%), grande parte declarou ser de cor de pele branca $(57,1 \%)$, o nível de escolaridade foi predominante no ensino médio completo $(56,1 \%)$, a maioria residia em zona urbana $(93,4 \%)$ e vivia com companheiro $(86,8 \%)$, quase a metade possuía renda familiar maior que três salários mínimos $(49,3 \%)$ (Tabela1).

Tabela 1: Caracterização socioeconômica, demográfica das mulheres internadas no período pós-parto, em uma maternidade de alto risco, Londrina, 2017.

\begin{tabular}{lcc}
\hline Variáveis socioeconômica e demográfica & $\mathrm{N}$ & $\%$ \\
\hline Idade & 26 & 8,1 \\
Menor que 19 anos & 154 & 48,2 \\
$19-29$ anos & 121 & 37,9 \\
30 - 39 anos & 18 & 5,6 \\
Maior que 39 anos & & \\
Cor de pele autodeclarada & 182 & 57,1 \\
Branca & 132 & 41,2 \\
Parda/Negra & 3 & 0,9 \\
Amarela & 2 & 0,6 \\
Indígena & &
\end{tabular}




\begin{tabular}{lcc} 
Ensino fundamental & 89 & 27,8 \\
Ensino médio & 179 & 56,1 \\
Ensino Superior & 49 & 15,3 \\
Analfabeta & 2 & 0,6 \\
Residência & 298 & 93,4 \\
Zona Urbana & 21 & 6,5 \\
Zona Rural & & \\
Situação conjugal & 277 & 86,8 \\
Com companheiro & 42 & 13,1 \\
Sem companheiro & & \\
Renda Familiar & 49 & 15,4 \\
Até 1 salário mínimo & 115 & 35,3 \\
De 2 a 3 salários mínimos & 155 & 49,3 \\
Mais de 3 salários mínimos & 319 & 100 \\
\hline Total & & \\
\hline
\end{tabular}

* Salário mínimo no ano de 2017- R \$937,00

Quanto à paridade, 40,4\% eram primíparas; 28,8\% secundíparas e 30,7\% multíparas. $\mathrm{O}$ intervalo entre um parto e outro que se destacou foi maior que dois anos $(52,97 \%)$. $\mathrm{O}$ estudo mostra que a maioria das mulheres passaram por cesariana $(60,8 \%)$, dessa forma, o local de parto mais frequente foi Centro Cirúrgico (CC). (Tabela 2)

Tabela 2: Caracterização segundo dados obstétricos, das mulheres internadas no período pós-parto, em uma maternidade de alto risco, Londrina, 2017.

\begin{tabular}{lcc}
\hline Dados obstétricos & $\mathrm{N}$ & $\%$ \\
\hline Número de gestações anteriores & 26 & 8,1 \\
Primípara & 154 & 48,2 \\
Secundípara & 121 & 37,9 \\
Multípara & 18 & 5,6 \\
Números de partos normais & & \\
1 & 182 & 57,1 \\
2 a 3 & 132 & 41,2 \\
4 ou mais & 3 & 0,9 \\
Não se aplica & 2 & 0,6 \\
Número de cesarianas & & \\
1 & 89 & 27,8 \\
2 a 3 & 179 & 56,1 \\
4 ou mais & 49 & 15,3 \\
Não se aplica & 2 & 0,6 \\
Intervalo Interpartal & & \\
< 1 ano & 298 & 93,4 \\
1 a 2 anos & 21 & 6,5 \\
> 2 anos & &
\end{tabular}


Não se aplica

Tipo de parto

Normal

Cesárea

Local do parto

49

15,4

Centro cirúrgico

115

35,3

Sala de parto

155

49,3

Quarto

Outros

6

1,8

Total

319

100

Quanto à informação sobre o direito ao acompanhante, 62,6\% das mulheres tiveram esse esclarecimento. No entanto, a quantidade de quem não recebeu informação ainda é significativa. Observa-se que foi mais frequente não receber a informação sobre o direito a um acompanhante no puerpério $(92,4 \%)$, no momento do parto $(82,1 \%)$, e na internação $(37,3 \%)$ (Tabela 3$)$.

Tabela 3: Caracterização da informação do direito ao acompanhante no momento da internação, do parto e do puerpério de mulheres internadas no período pósparto, em uma maternidade de alto risco, Londrina, 2017.

\begin{tabular}{lcc}
\hline $\begin{array}{l}\text { Momento da informação relacionada ao } \\
\text { direito ao acompanhante }\end{array}$ & $\mathrm{N}$ & $\%$ \\
\hline Internação & 119 & 37,3 \\
Sim & 200 & 62,7 \\
Não & & \\
Parto & 57 & 17,8 \\
Sim & 262 & 82,1 \\
Não & & \\
Puerpério & 24 & 7,5 \\
Sim & 295 & 92,4 \\
Não & & \\
Acompanhante presente no trabalho & & \\
de parto e parto & 121 & 37,9 \\
Sim & 198 & 62,0 \\
Não & 319 & 100 \\
\hline Total & & \\
\hline
\end{tabular}

No momento do parto $62,0 \%$ das parturientes não tiveram acompanhante, os motivos foram, não terem sido informadas $(66,1 \%)$; rotina hospitalar desfavorável ao acolhimento do acompanhante (23,2\%); dificuldades em relação a dinâmica familiar (6,0\%), e intercorrências maternas e/ou neonatais (4,5\%) (Tabela 4). 
Tabela 4: Distribuição dos motivos pelos quais as mulheres não tiveram acompanhante no trabalho de parto e parto, em uma maternidade de alto risco, Londrina, 2017.

\begin{tabular}{lcc}
\hline Por que não teve acompanhante & $\mathrm{N}$ & $\%$ \\
\hline Dificuldades em relação a dinâmica familiar & 12 & 6,0 \\
Intercorrências maternas e/ou neonatais & 9 & 4,5 \\
Dificuldades em relação a rotina hospitalar & 46 & 23,2 \\
Não foi informado o motivo & 131 & 66,1 \\
\hline Total & 198 & 100 \\
\hline
\end{tabular}

\section{DISCUSSÃO}

O perfil socioeconômico e demográfico predominante no estudo, foram: mulheres jovens adultas; com nível de escolaridade acima da média nacional; com companheiro; renda maior que três salários mínimos; residindo na área urbana e cor de pele branca. Descrição que difere da literatura, pois estudos apontam que as características individuais e sociodemográficas desfavoráveis contribuem para o risco gestacional. Neste estudo mesmo com a discrepância de perfil contrapondo aos achados da literatura, as participantes desenvolveram situações que as classificassem como gestantes de alto risco ${ }^{8-9-10}$.

As gestações classificadas como de alto risco, tem o encaminhamento para conclusão da gestação em instituições de alta complexidades, com o intuito de prevenir e/ou atender qualquer tipo de emergência que pode ocorrer ao binômio8. A pesquisa identificou que a conclusão da gestação, em sua maioria, foi por meio da cesariana, transpondo a taxa ideal preconizada pelo Ministério da Saúde de até 30\%. Embora as gestantes desse estudo sejam de alto risco, a OMS enfatiza que o parto cesáreo deve ser realizado baseado em suas indicações $^{11-12-13}$.

Mesmo o tipo de parto sendo cesárea, a lei do acompanhante também é válida3. No presente estudo, mais da metade das pacientes foram informadas sobre o direito ao acompanhante, sendo que o momento da informação foi mais frequente na internação. No entanto, considera-se o pré-natal o melhor momento para orientação sobre o direito do acompanhante, em uma pesquisa onde os acompanhantes foram orientados durante o prénatal, as parturientes tiveram o sentimento de segurança durante o parto, e além do apoio emocional, conseguiram realizar outras ações, com aparente orgulho pela execução ${ }^{14-15}$. 
Embora essas mulheres tenham sido informadas sobre a lei do acompanhante na internação, um número significativo de parturientes não teve acompanhantes no parto e entre os motivos, a dificuldade em relação a rotina hospitalar fio relevante. Corroborando com a literatura em um estudo realizado com 83 mulheres, onde 58\% não tiveram acompanhante, porém $90 \%$ das puérperas desejava ter um acompanhante e entre os motivos de não terem tido esta oportunidade, $17 \%$ relataram o impedimento pela equipe de saúde ${ }^{16}$.

Para os profissionais de saúde, os obstáculos para cumprir integralmente a Lei do Acompanhante estão relacionados com ideias pré-concebidas de que o ambiente hospitalar não é lugar para o acompanhante e que sua presença teria influência negativa na organização do processo de trabalho. Esse discurso revela que o modelo de atenção à saúde ainda é biologicista, apesar das políticas de humanização ${ }^{17}$.

A participação do acompanhante no parto e nascimento é importante, pois está relacionada com a minimização do sentimento de solidão e de dor. A presença de alguém de confiança para a mulher proporciona conforto e calma a elas, fazendo com que se sintam mais $\operatorname{seguras}^{4-18}$.

\section{CONSIDERAÇÕES FINAIS}

Notam-se dificuldades para incorporar o acompanhante na rotina e no atendimento ao trabalho de parto, parto e puerpério de mulheres classificadas como de alto risco, na instituição em estudo. Evidenciou uma lacuna na pratica da lei no 11.108 de 2005, decorrente da rotina hospitalar e até mesmo do modelo usado para disseminar a informação sobre o direito ao acompanhante, essas situações podem estar relacionadas tanto ao espaço físico, como com a formação profissional, ainda biologicista, em que as práticas humanizadas encontram barreiras para serem efetivadas, assim como o número limitado de profissionais no acolhimento ao acompanhante e assistência ao paciente.

É necessário que os serviços se adequem para receber os acompanhantes e além disso, possam integrá-los à rotina de atendimento à parturiente de alto risco, promovendo atenção mais humanizada e viabilizando os benefícios dessa prática para mãe e recémnascido. 


\section{REFERÊNCIAS}

1. GRILO, Carmen Simone; D‘ORSI, Eleonora; DOMINGUES, Rosa Maria Soares Madeira; TORRES, Jacqueline Alves; DIAS, Marcos Augusto Bastos; SCHNECK , Camilla A., et al. Implementação da presença de acompanhantes durante a internação para o parto: dados da pesquisa nacional Nascer no Brasil. Cadernos de Saúde Pública, v.30, n.1, p. 140-53, 2014.

2. FONTE, Danyelle Oliveira; MONTEFUSCO, Selma Rodrigues Alves. A importância da presença do acompanhante junto a parturiente e seu bebê. Revista Científica da Escola de Saúde Pública. v.2, n.3, p.127-36, 2017.

3. Brasil. Presidência da República, Subchefia para Assuntos Jurídicos. Lei Federal $n^{\circ}$ 11.108, de 07 de abril de 2005. Altera a lei 8.080, de 19 de setembro de 1990, para garantir as parturientes o direito a presença de acompanhante durante o trabalho de parto, parto e pós-parto imediato, no âmbito do Sistema Único De Saúde - SUS. Brasília, DF; 2005.

4. DODOU, Hilana Dayana; RODRIGUES, Dafne Paiva; GUERREIRO, Eryjosy Marculino; GUEDES, Maria Vilani Cavalcante; LAGO, Pamela Nery; MESQUITA, Nayara Sousa. The contribution of the companion to the humanization of delivery and birth: perceptions of puerperal women. Escola Anna Nery - Revista de Enfermagem. v.18, n.2, p.262-69, 2014.

5. SILVA, Andréa Lorena Santos; NASCIMENTO, Enilda Rosendo; COELHO, Edméia de Almeida Cardoso. Nurses practices to promote dignity, participation and empowerment of women in natural childbirth. Escola Anna Nery - Revista de Enfermagem. v.19, n.3, p. 424-31, 2015.

6. MONGUILHOTT, Juliana Jacques da Costa; BRUGGEMANN, Odaléa Maria; FREITAS, Paulo Fontoura; D'ORSI, Eleonora. Nascer no Brasil: a presença do acompanhante favorece a aplicação das boas práticas na atenção ao parto na região Sul. Revista de Saúde Pública. v. 52, p. 1-11, 2018.

7. BARBETA, Pedro Alberto. Fórmula para o cálculo do tamanho mínimo da amostra. Estatística aplicada às ciências sociais. 8. ed. Florianópolis: Editora da UFSC; 2012.

8. Ministério da Saúde (BR). Secretaria de Atenção à Saúde. Departamento de Ações Programáticas Estratégicas. Gestação de alto risco: manual técnico. Brasília; 2012.

9. SANTOS, Bruna Teixeira dos; MONTEIRO, Bruna Karoline Santos Melo; SANTOS, José Gilmar Costa; SILVA, Renata Sousa de Santana; LEMOS, Lígia Mara Dolce de; DIAS, Júlia Maria Gonçalves Socioeconomic conditions, gestacional risk and importance of relationship between obstetrician and high risk pregnant women. Revista de Enfermagem da Ufpi. v.3, n.5, p.36-41, 2016. 
10. Instituto Brasileiro de Geografia e Estatística. Pesquisa Nacional por Amostra de Domicílios Contínua. Rio de Janeiro: IBGE, 201.

11. Ministério da Saúde (BR). Gabinete do Ministro. Portaria $\mathrm{n}^{\circ} 1.020$, de 29 de maio de 2013. Diário Oficial da União [DOU]. Brasília, 31 de maio de 2013; Seção 1, n.103, p. 72.

12. Ministério da Saúde (BR). Comissão nacional de incorporação de tecnologias no SUS. Diretrizes de Atenção à Gestante: a operação cesárea. Brasília, 2015.

13. Organização das Nações Unidas. Declaração da OMS sobre taxas de cesáreas. Suiça: OMS. 2015.

14. BRUGGEMANN, Odaléa Maria; EBSEN, Erika Simas; EBELE, Romana Raquel; BATISTA, Bruna Daniela. Possibilidades de inserção do acompanhante no parto nas instituições públicas. Ciência \& Saúde Coletiva. v. 21, n.8, p. 2555-64, 2016.

15. SOUZA, Marli Aparecida Rocha de; WALL, Marilene Loewen; THULER, Andréa Cristina de Morais Chaves; FREIRE, Márcia Helena de Souza; SANTOS, Evangelia Kotzias Atherino dos.Vivência do acompanhante da parturiente no processo de parto. Revista de Enfermagem Ufpe Online v. 3, n. 12, p.626-34, 2018.

16. VENDRÚSCOLO, Claudia Tomasi; KRUEL, Cristina Saling. Livre escolha da parturiente pela acompanhante e seus entraves: desafios para a humanização da assistência ao parto e nascimento. n.49; p. 52-70, 2017.

17. BRUGGEMANN, Odaléa Maria; EBELE, Romana Raquel; EBSEN, Erika Simas; BATISTA, Bruna Daniela. No parto vaginal e na cesariana acompanhante não entra: discursos de enfermeiras e diretores técnicos. Revista Gaúcha de Enfermagem. v. 36, p. $152-5,2015$.

18. SANTOS, Ana Lívia Santana; OLIVEIRA, Aline Reis Souza de; AMORIM, Torcata; SILVA, Uanisléia Lima da. O acompanhante no trabalho de parto sob a perspectiva da puérpera. Revista de Enfermagem da Ufsm. v.5, n.3, p.531-40, 2015. 